\title{
Taxonomic studies on subgenus Pseudomegachile Friese under genus Megachile Latreille (Hymenoptera) from Sutlej basin plains (India)
}

\author{
Priyanka Kumari* \\ High Altitude Regional Centre, Zoological Survey of India, Solan-173211 (HP), India \\ Neelima R. Kumar \\ Department of Zoology, Panjab University, Chandigarh-160014 (UT) India \\ Avtar K. Sidhu \\ High Altitude Regional Centre, Zoological Survey of India, Solan-173211 (HP), India \\ *Corresponding author. E-mail: priyanka.dadhwal.chandel@gmail.com
}

\begin{abstract}
Genus Megachile forms an integral part of the ecosystem as its members are important pollinators of both wild and cultivated flora. Surveying on a large scale helps in gathering a lot of information about their spatial and temporal data. This also helps in studying their biodiversity and taxonomy. Three species of solitary bees under subgenus Pseudomegachile Friese of the genus Megachile Latreille collected from Sutlej basin plains of India have been studied and described taxonomically. Megachile (Pseudomegachile) creusa (Bingham), $M$. (P.) ramakrishnae Cockerell and $M$. (P.) elfrona (Cameron) with 21 examples are described including their morphological characters, taxonomically important characters, zoogeographic records, floral associations along with genitalic and sternal plates. Since most of the areas included in the study have been surveyed first time for Megachile study, these are new records for the studied area.
\end{abstract}

Keywords: Pseudomegachile, Male genitalia, Sterna, Morphology

\section{Article Info}

https://doi.org/10.31018/

jans.vi.2259

Received: April 26, 2020

Revised: May 12, 2020

Accepted: May 20, 2020

\section{How to Cite}

Kumari, P. et al. (2020).

Taxonomic studies on subgenus Pseudomegachile Friese under genus Megachile Latreille (Hymenoptera) from Sutlej basin plains (India). Journal of Applied and Natural Science, 12(2): 146 - 155 https://doi.org/10.31018/ jans.vi.2259

\section{INTRODUCTION}

Systematic classification of this group includes family Apidae, tribe Megachilini and genus Megachile Latrellie. Around 1520 species of Megachile were reported worldwide in 53 subgenera (Michener, 2007; Raw, 2007; Praz, 2017; Kumari, 2019) with a total of 75 species reported from India (Gupta, 1999). Diversity of tribe Megachilini is very limited. Two out of three genera of this tribe have been reported from India, i.e. Megachile and Coelioxys Latrielle. A total of 56 subgenera have been reported from all over the world in genus Megachile and 17 subgenera from India (Michener, 2000). In the present study subgenus Pseudomegachile is being studied and reported from Sutlej basins in India. The bees belonging to subgenus Pseudomegachile are more frequent in these plains with fusco-rufous legs in some species and clearly banded tergal fasciae abdomen in others. These are fast fliers while foraging in general, thus aiding a lot in pollination. These bees have to make many rounds to the flowers as the collection of pollen in their brush is not enough as compared to corbicula of Apis species, the species richness of the species of subgenus Pseudomegachile has been directly proportional to the presence of preferred flora. Therefore, the present study was undertaken to survey and study the zoogeographic distribution and diversity of the subgenus Pseudomegachile from the Sutlej basin of Northern plains. The members of the subgenus were identified, classified and studied taxonomically.

\section{MATERIALS AND METHODS}

Collection forms the most important step in the taxonomic studies. The study area comprised the Sutlej basin plains which include Punjab, Haryana and Chandigarh (U.T.). The various areas covered in present studies are Parwanoo $\left(30.8372^{\circ}\right.$ $\left.\mathrm{N}, 76.9614^{\circ} \mathrm{E}\right)$ in Himachal Pradesh; Patiala $\left(30.3398^{\circ} \mathrm{N}, 76.3869^{\circ} \mathrm{E}\right)$, Amritsar $\left(30.6942^{\circ} \mathrm{N}\right.$, $\left.76.8606^{\circ} \mathrm{E}\right)$, Una $\left(31.4684^{\circ} \mathrm{N}, 76.2708^{\circ} \mathrm{E}\right)$ and Jalandhar $\left(31.3260^{\circ} \mathrm{N}, 75.5762^{\circ} \mathrm{E}\right)$ in Punjab and Panchkula $\left(30.6942^{\circ} \mathrm{N}, 76.8606^{\circ} \mathrm{E}\right)$, Hisar $\left(29.1492^{\circ} \mathrm{N}, 75.7217^{\circ} \mathrm{E}\right)$ in Haryana (Fig. 1). The collection has been done during bright sunny days with the help of sweeping nets while bees were foraging on the flowers. Examples collected were transferred to charged collection bottle. After the bees were killed, stretched and properly preserved in fumigated insect boxes. Keys given by 
Bingham (1897), Michener (2000, 2007) have been followed to identify the species. Canon D60 digital camera has been used to photograph the adult examples. After dissections, genitalic slides and sternal slides were prepared and photographed with Leica microscope at 20X. A total of 37 characters were finalized for the morphological measurements (Gupta, 1999 and Bzydk, 2012). Measurements were made in $\mathrm{mm}$ with Radical Stereozoom microscope (model RSM 9 fitted with software Progres Capture Pro version 2.1.1 and CT5 Jenoptik camera (Table 1).

\section{OBSERVATIONS AND DISCUSSION}

\section{Genus Megachile Latreille}

Megachile Latreille 1802, Histoire Naturelle des Fourmis, Vol. II, p.43.

Type species: Apis centuncuralis Linnaeus Anthophora Fabricius 1804, Mitt.derDeutsc. Entomol.Gesells. Vol. X, p. 372.

Megachile Smith 1853, Cat. Hym.Vol. I, p. 149.

Megachile Dalla Torre 1894, Cat. Hym. Vol. X, p. 417.

Megachile Bingham 1897, Fauna of British India. Vol. I, p. 470

Diagnosis: Bees included in this genus varies a lot in their morphology, size and behaviour. They are non-metallic black bees with cordate abdomen and tergal fasciae. In females pollen brush is present on underside of abdomen.

\section{Subgenus Pseudomegachile Friese}

Pseudomegachile Friese 1899, "Die BinenEuropas". Vol. 5, p. 36.

Type species: Megachile erectorum Lepeltier Archimegachile Alfken 1933, Konowia. Vol. 12, p. 243.

Pseudomegachile Pasteels 1965, Ann. Mus. R. Afr. Centr. Tervurn Sci. Zool. Vol. 137, p. 277.

Diagnosis : Clypeal margin broadly in bent or transverse in profile in females but broadly outcurved in males; mandible 4 dentate in females and obscurely 4 dentate in males; spinose carina of tergum 6 in males; dense hairs on sternum 6 , fringed margin of sternum 8 , apex rounded; dense brush of plumose hairs on inner sides of gonostipites close to apex. Dichotomous keys were selfdeveloped for the identified species.

Key to the species of subgenus Pseudomegachile Friese

Pubescence all over the body snow white, legs may or may not be reddish brown.......2

Pubescece on head, thorax and at least first two segments of abdomen fulvous...........4

Legs fully reddish orange in both sexes................. creusa Bingham

Legs not fully reddish orange............3

Abdomen parallel sided, broad and short in males; apices of mid hind legs covered with fulvous dense pubescence present on paraocular areas, first two tergal fasciae reddish brown rest with

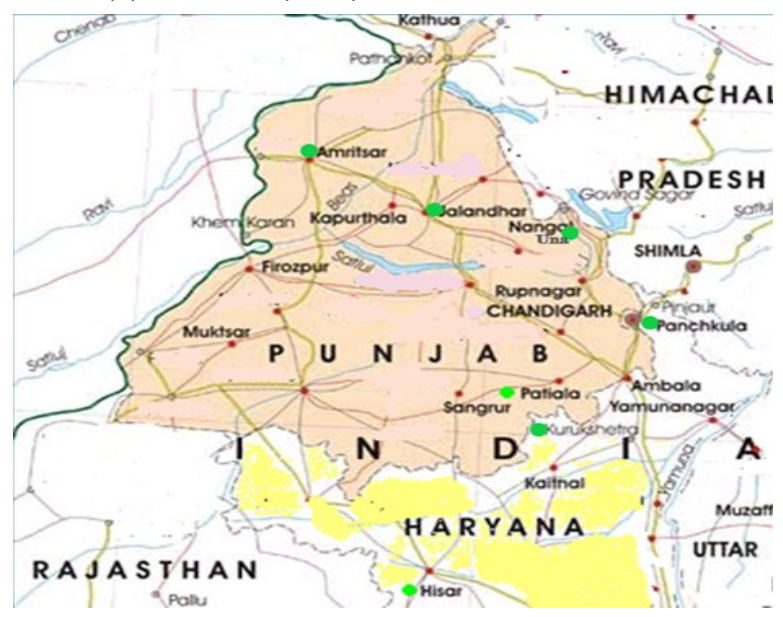

Fig. 1. Map of Sutlej basin of northern plains showing the collection areas.

white pubescence...............buddhae Bingham

Abdomen cordate, tapering at apex; apices of tarsi of mid and hind legs dull white, line missing on scutum, all tergal fasciae with white pubescence .....elfrona Cameron

Megachile (Pseudomegachile) creusa Bingham, 1898

Megachile creusa Bingham 1898, Jour. Bomb. Nat. Hist. Soc. Vol. XII, p. 125.

Female (Plate 1, Figs. a-f)

Diagnosis: Integument black, flagellum and mandible tip ferruginous; legs orange red; claws black. Pubescence on face, gena, lateral sides of thorax, basal fasciae of abdomen niveous; scopal bristles ochroleucous; tarsi dorsally white.

Head: Clypeus outcurved, sculptured finely, apical margin inflated and smooth; supraclypeus imperceptibly bulging, punctate finely; lateral ocelli near each other than occipital margin; laterally genal width more than the eye; five dentate mandibles.

Mesosoma: Scutum arched, convex, punctate; lateral extensions of metanotum not depressed, punctate; wings subfuscous at apex; Posterior margin of scutellum oval and covered with dense erect small white hairs; legs fuscorufous, apicodorsally spine present on tibiae, claws with bristles present.

Metasoma: Metasoma broad, tapering in profile; incarinate basal concavity margin; pregradular area partially exposed on terga $2-5$, grooved shallowly on terga $4-5$, tergum $6^{\text {th }}$ concave in profile; sterna slightly convex in profile, sternal fasciae widely interrupted medially, sternum $6^{\text {th }}$ without dense fringe of hairs.

Male (Plate 2, Figs. a-i)

Diagnosis: Integument black, legs fuscorufous; pubescence on face, supraclypeus, gena, dorsal sides of tibiae, scutellum margins, fasciae on terga and sterna with different shades of white.

Head: Clypeus bulging, smooth outcurved margin; supraclypeus convex and punctured closely; clypeus, supraclypeus, hypostome, paraocular area 
Kumari, P. et al. / J. Appl. \& Nat. Sci. 12(2): 146 - 155 (2020)

Table 1. Measurements $(\mathrm{mm})$ of the species belonging to subgenus Pseudomegachile Friese.

\begin{tabular}{|c|c|c|c|c|c|c|c|}
\hline S. No. & Characters & $\begin{array}{l}\text { Magnifica- } \\
\text { tion }\end{array}$ & M. CI & $\begin{array}{r}\text { eusa } \\
0\end{array}$ & $\begin{array}{c}\text { M. elfrona } \\
\text { o }\end{array}$ & M. budhae & \\
\hline 1. & $\mathrm{BdL}$ & $4 X$ & 12.6 & 9.21 & 11.22 & 13.2 & \\
\hline 2. & $\mathrm{HdL}$ & $10 x$ & 3.89 & 3.84 & 3.61 & 4.08 & \\
\hline 3. & $\mathrm{HdB}$ & $10 x$ & 2.98 & 2.77 & 2.89 & 3.45 & \\
\hline 4. & $\mathrm{laD}$ & $10 x$ & 0.91 & 0.89 & 0.85 & 1.03 & \\
\hline 5. & $A n D$ & $10 x$ & 1.3 & 1.46 & 1.37 & 1.47 & \\
\hline 6. & AoAsD & $10 x$ & 1.07 & 0.92 & 1.05 & 1.24 & \\
\hline 7. & UcD & $10 x$ & 2.06 & 2.18 & 1.53 & 2.31 & \\
\hline 8. & LcD & $10 x$ & 1.99 & 1.94 & 1.98 & 2.01 & \\
\hline 9. & $\mathrm{VW}$ & $10 x$ & 1.70 & 1.81 & 1.12 & 1.54 & \\
\hline 10. & $\mathrm{CbW}, \mathrm{CaW}$ & $20 x$ & $\begin{array}{l}0.59 \\
1.55\end{array}$ & $\begin{array}{l}1.02 \\
1.46\end{array}$ & $\begin{array}{l}0.81 \\
1.30\end{array}$ & $\begin{array}{l}0.75 \\
1.25\end{array}$ & \\
\hline 11. & $\mathrm{CmL}$ & $20 x$ & 1.52 & 1.55 & 1.20 & 1.17 & \\
\hline 12. & LL & $20 x$ & 1.98 & 0.96 & 1.78 & 1.02 & \\
\hline 13. & WL & $20 X$ & 2.23 & 1.09 & 2.10 & 1.88 & \\
\hline 14. & Ms & $20 x$ & .120 & 0.10 & 0.12 & 0.05 & \\
\hline 15. & $\mathrm{MnL}$ & $20 x$ & 1.52 & 1.18 & 1.35 & 1.55 & \\
\hline 16. & ScL & $20 x$ & 0.74 & 0.68 & 0.69 & 0.67 & \\
\hline 17. & $\mathrm{PdL}$ & $20 x$ & 0.12 & 0.20 & 0.11 & 0.27 & \\
\hline 18. & $A n L$ & $20 x$ & $\begin{array}{ll}0.12, & 0.22, \\
0.15, & 0.20, \\
0.23, & 0.24, \\
0.21, & 0.21, \\
0.34 & \end{array}$ & $\begin{array}{ll}0.18, & 0.15 \\
0.26, & 0.17 \\
0.18, & 0.21, \\
0.23, & 0.11, \\
0.23, & 0.23\end{array}$ & $\begin{array}{l}0.15, \\
0.24,0.23,0.28 \\
0.23,0.29,0.27 \\
0.25,0.27,0.26 \\
0.39\end{array}$ & $\begin{array}{l}0.17,0.27 \\
0.29, \quad 0.31 \\
0.28, \quad 0.30 \\
0.25,0.45\end{array}$ & $\begin{array}{l}0.28 \\
0.32 \\
0.29\end{array}$ \\
\hline 19. & AnW & $20 x$ & $0.24,0.20$ & $0.19,0.21$ & $0.21,0.15$ & $0.24,0.25$ & \\
\hline 20. & FwL & $7.5 X$ & 8.72 & 5.68 & 6.39 & 8.95 & \\
\hline 21. & McL & $7.5 X$ & 2.18 & 1.49 & 1.65 & 2.29 & \\
\hline 22. & ScL & $7.5 X$ & 0.73 & 0.45 & 0.52 & 0.54 & \\
\hline 23. & $\mathrm{HwL}$ & $7.5 X$ & 6.17 & 4.17 & 5.32 & 6.35 & \\
\hline 24. & JIL & $7.5 X$ & 2.02 & .82 & 1.36 & 1.18 & \\
\hline 25. & VIL & $7.5 X$ & 3.28 & 2.36 & 2.47 & 2.53 & \\
\hline 26. & ScL & $7.5 X$ & 3.4 & 2.16 & 2.12 & 2.87 & \\
\hline 27. & ScW & $7.5 X$ & 3.27 & 2.98 & 2.97 & 4.01 & \\
\hline 28. & SuL & $7.5 X$ & .99 & .96 & 1.61 & 2.46 & \\
\hline 29. & MsL & $7.5 X$ & 3.80 & 3.91 & 3.71 & 4.45 & \\
\hline 30. & TgW & $7.5 X$ & $\begin{array}{l}3.53,3.76 \\
3.80,3.36 \\
2.94,1.23\end{array}$ & $\begin{array}{l}2.96,3.34 \\
3.40,3.23 \\
2.47,1.25\end{array}$ & $\begin{array}{l}2.95,3.36 \\
3.17,2.87 \\
2.38,1.44\end{array}$ & $\begin{array}{l}4.30,4.46 \\
4.09,3.31 \\
2.45,1.34\end{array}$ & \\
\hline 31. & MtL & $7.5 X$ & 6.03 & 4.16 & 4.89 & 5.11 & \\
\hline 32. & $\mathrm{CxL}$ & $7.5 X$ & 0.84 & 0.88 & 0.55 & 1.17 & \\
\hline 33. & TrL & $7.5 X$ & 1.45 & 0.42 & 0.61 & 0.78 & \\
\hline 34. & $\mathrm{FmL}$ & $7.5 X$ & 0.33 & 1.65 & 0.99 & 1.44 & \\
\hline 35. & TbL & $7.5 X$ & 2.07 & 1.37 & 1.12 & 2.59 & \\
\hline 36. & BtL & $7.5 X$ & 1.54 & 1.27 & 1.15 & 1.28 & \\
\hline 37. & DtL & $7.5 X$ & 0.52 & 0.43 & 0.40 & 0.90 & \\
\hline
\end{tabular}

Abbreviations: Body length:Bdl; HEAD: Head width -HdW, Head length -HdL, Interantennal distance- laD, Antennocular distance- AnD, Distance from anterior ocellus to antennal socket- AoAsD, Distance between upper inner margins of compound eyes- UcD, Distance between lower margins of compound eyes- LcD, Width of vertex- VW, Clypeus basal \& apical width- CbW, CaW, Clypeus median length- CmL, Length of labrum-LL, Width of labrum- WL, Malar space- Ms, Length of mandible- MnL; ANTENNAE: Length of scape- ScL, Length of pedicel-PdL, Length of segments of antennae- AnL, Width of segment vi \& x/xi-AnW; WINGS: Total length of fore wing-FwL, Marginal cell length- McL, Stigma cell length- ScL, Total length of hind wing- HwL, Length of jugal lobe- JIL, Length of vannal lobe- VIL; MESOSOMA: Median length of scutum- ScL, Maximum width of scutum- ScW, Median length of scutellum- SuL, Length of mesosoma- MsL; METASOMA: Relative width of terga 1-6- TgW, Length of metasoma- MtL; HIND LEG: Length of coxa- CxL, Length of trochanter- TrL, Length of femur- FmL, Length of tibia- TbL, Length of basitarsus- BtL, Length of distitarsus- DtL 
Kumari, P. et al. / J. Appl. \& Nat. Sci. 12(2): 146 - 155 (2020)
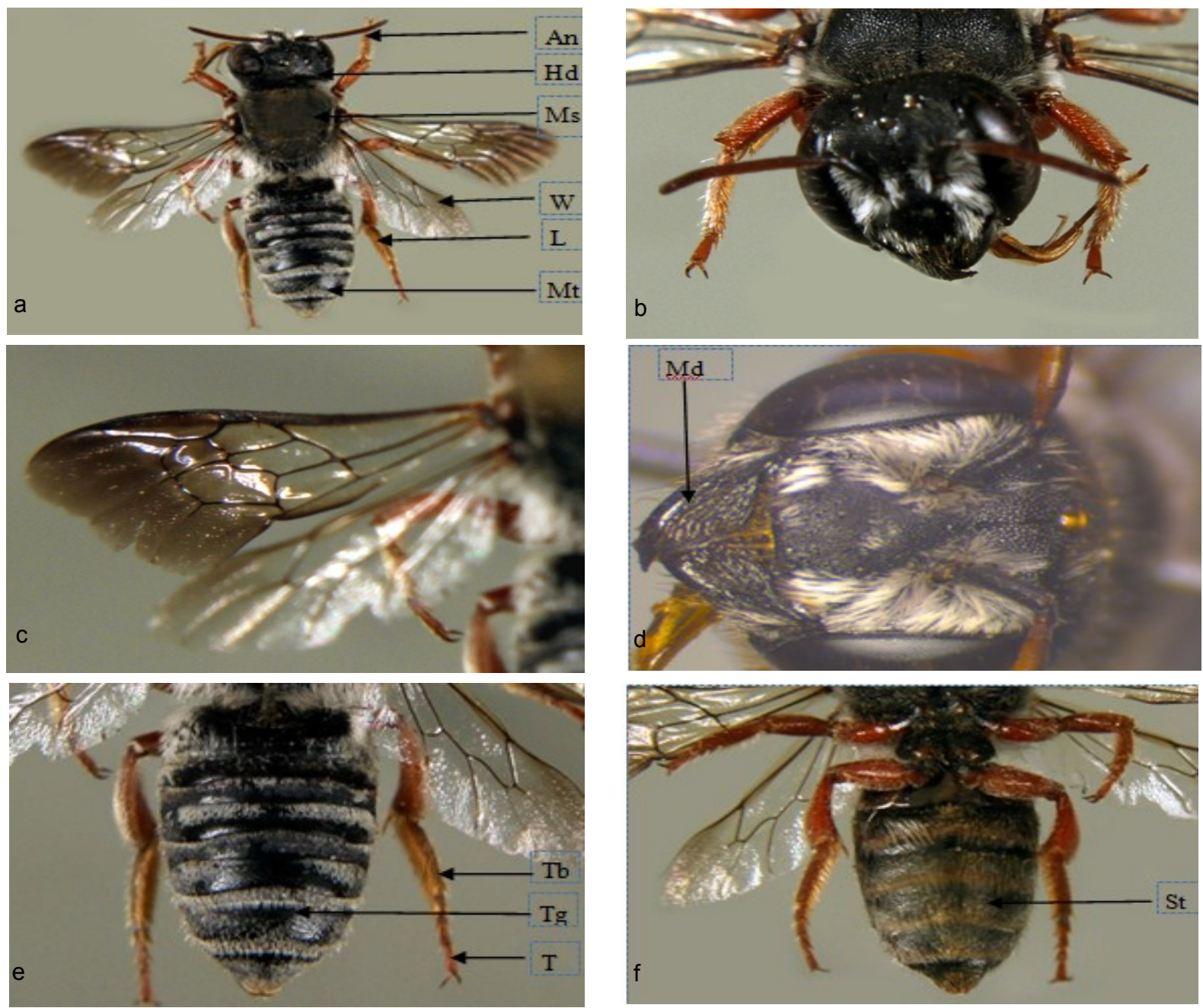

Plate 1. Megachile creusa (Bingham) female (a) Habitus, dorsal view (b) Head and Mesosoma, dorsal view (c) Wing (d) Mandibles and clypeus (e) Metasoma, dorsal view (f) Sterna, ventral view.

covered with white down; vertex black with scattered white down, sculptured coarsely; mandibles 3 dentate.

Mesosoma: Scutum raised with dense fuscopisceous and white down; scutellum mottled with posterior margin covered with cinereous down; wings hyaline, fuscous at apex.

Metasoma: Abdomen short, parallel sided, white apical fasciae; coxal spine erect, legs fuscotestaceous, apices of tarsi of legs excavated apically, white pubescence; exposed sterna with complete fasciae covered with white down.

External genitalia: Gonocoxite broad at base, narrows slowly to thin gonostylus covered all along with thick black hairs. Penis valves also thick at base and narrow at the apex. Apodemes of penis valves short and thick in gonobase.

Material examined: $2 \hat{\jmath}, 22 . i x .2014$, Patiala $\left(30.3398^{\circ} \mathrm{N}, 76.3869^{\circ} \mathrm{E}\right) ; 1$ ㅇ, 1.x.2014, Panchkula $\left(30.6942^{\circ} \mathrm{N}, 76.8606^{\circ} \mathrm{E}\right) ; 1$ \%, 11. iv. 2015, Panchkula $\left(30.6942^{\circ} \mathrm{N}, 76.8606^{\circ} \mathrm{E}\right) ; 2 \widehat{O}^{\hat{\sigma}}$, 10.v.2015, Amritsar (30.6942 $\left.{ }^{\circ} \mathrm{N}, 76.8606^{\circ} \mathrm{E}\right)$.
Distribution in India: Gujarat, Rajasthan, Punjab, Haryana, Uttarakhand.

Distribution elsewhere: North Africa, Iran, Pakistan, Afghanistan, Western China, Nepal.

Floral associations (Plate A): Fabaceae: $\mathrm{Ca}$ janus cajan (L.), Tephrosia hamiltonii J., Doliches lablabL.; Brassicaceae: Lobularia maritima (L.)

Remarks: Its taxonomic position has not much changed since its original description by Bingham in 1898. It was described as $M$. creusa.

\section{Megachile (Pseudomegachile) buddhae Dalla} Torre, 1894

Megachile budhae Dalla Torre 1894, Cat. Hym. Vol. X., p. 423.

Megachile rufipes Smith 1858, Jour. Linn. Soc. Vol. XVIII, p. 177.

Megachile femorata Bingham 1897, Fauna of Brit. Ind. Vol. I, p. 488.

Male (Plate 3, Figs. a-h)

Diagnosis: Integument black; flagellum, legs with redness; claws tips black. Pubescence on face, gena, thorax, first two abdominal segments ful- 
Kumari, P. et al. / J. Appl. \& Nat. Sci. 12(2): 146 - 155 (2020)
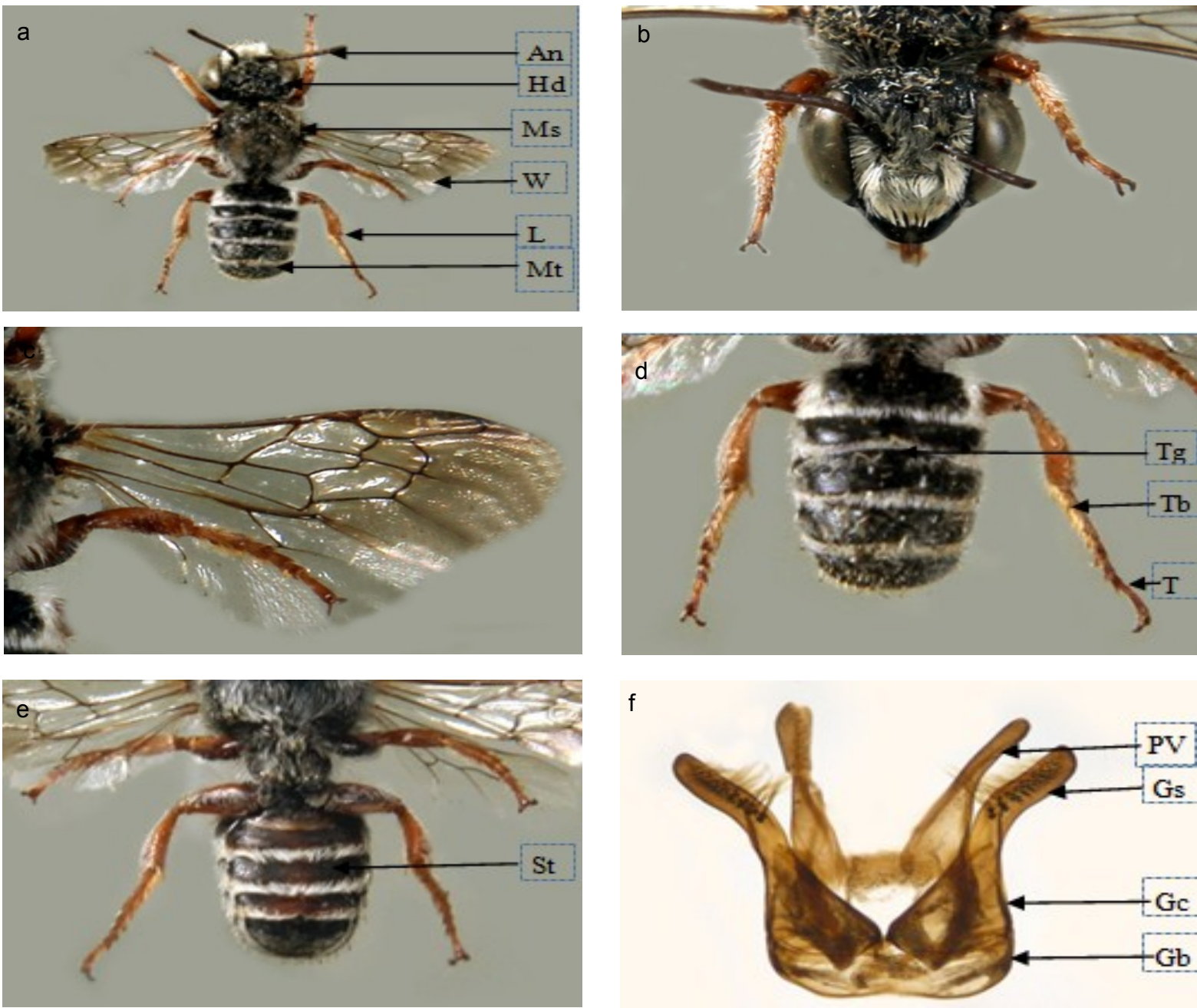

f
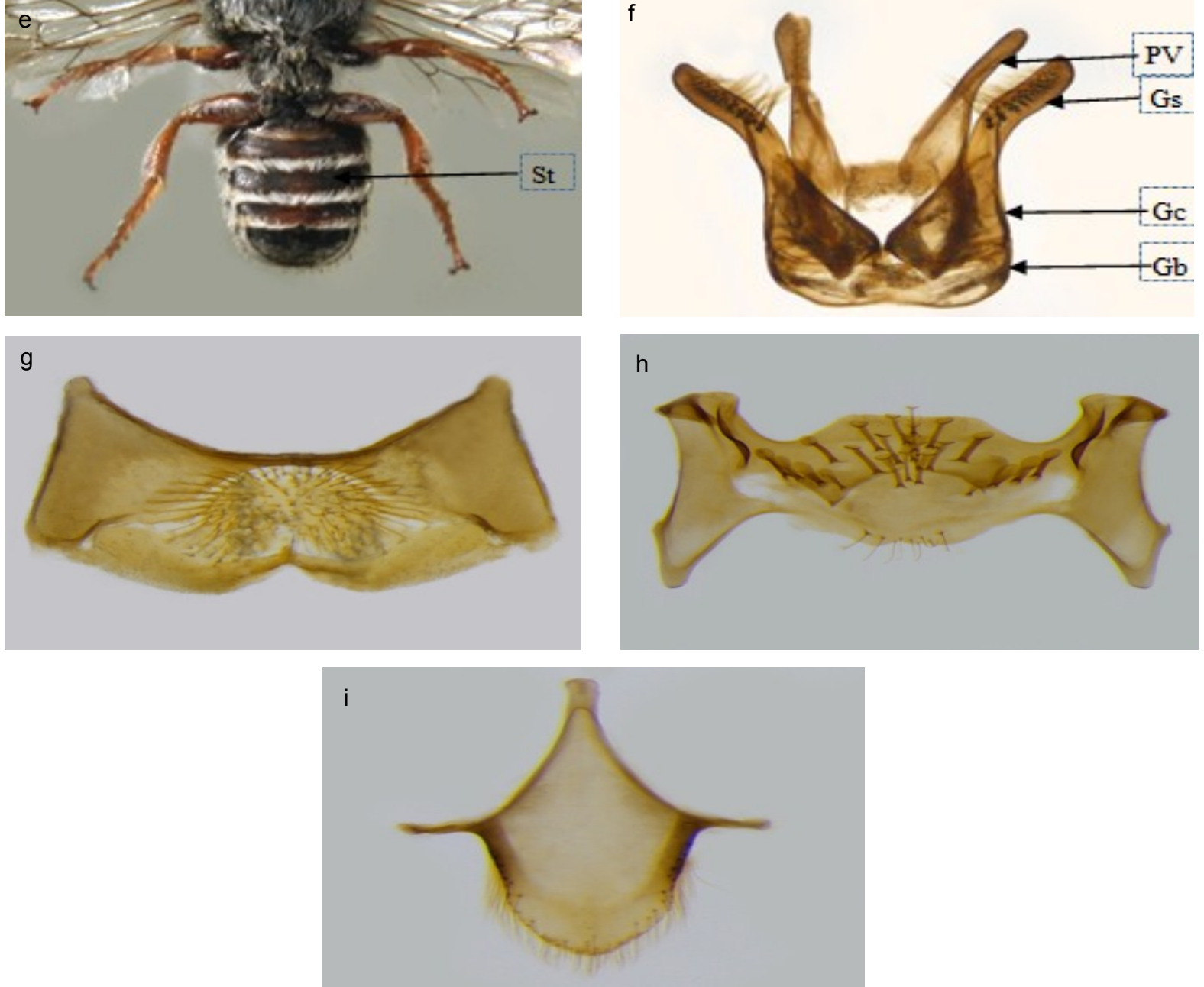

Plate 2. Megachile creusa (Bingham) male (a) Habitus, dorsal view (b) Head and Mesosoma, dorsal view (c) Wing (d) Metasoma, dorsal view (e) Sterna, ventral view (f) Genitalia (g) Sternum 5 (h) Sternum 6 (i) Sternum 8. 


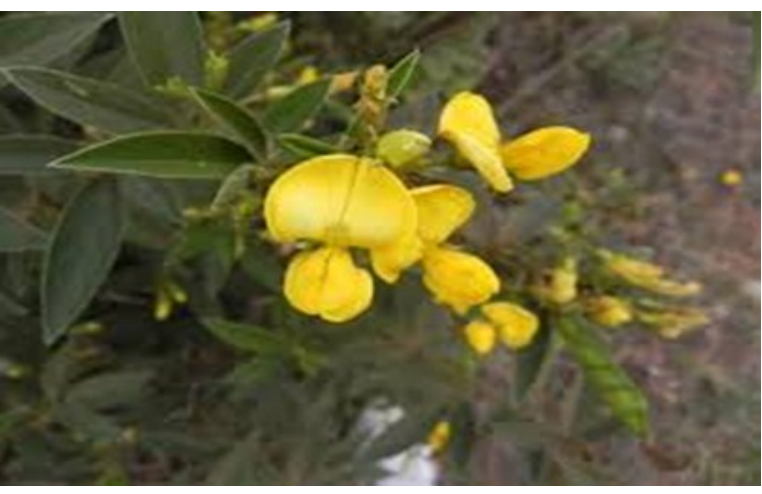

Cajanus cajan (L.)

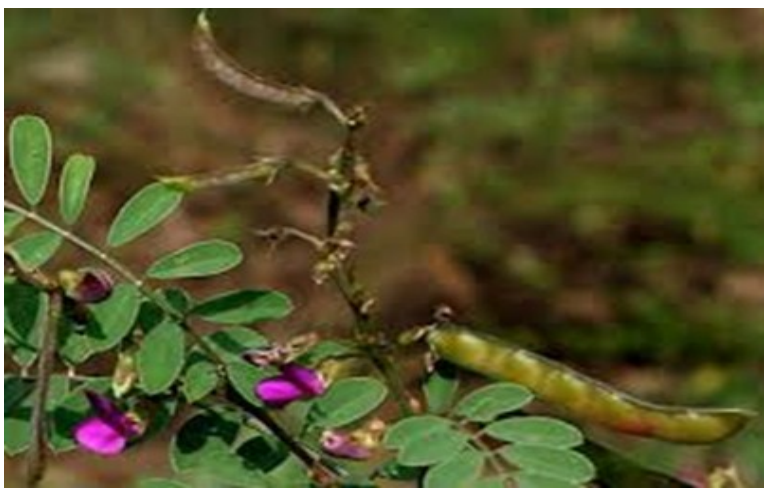

Doliches lablab L.

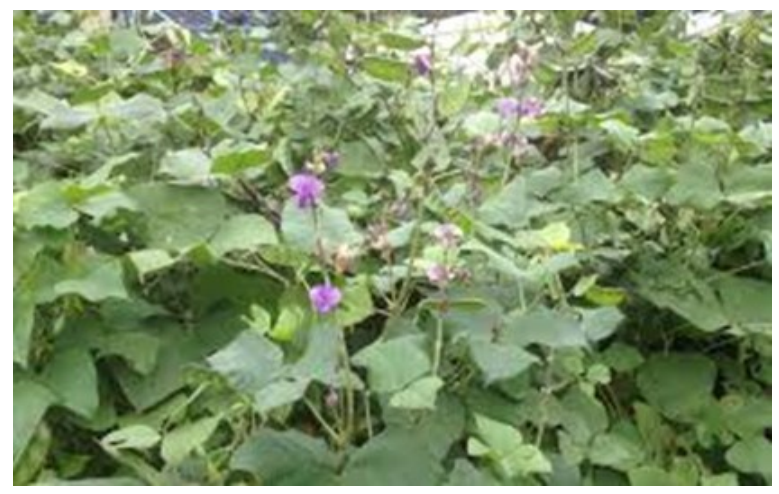

Tephrosia hamiltonii J.

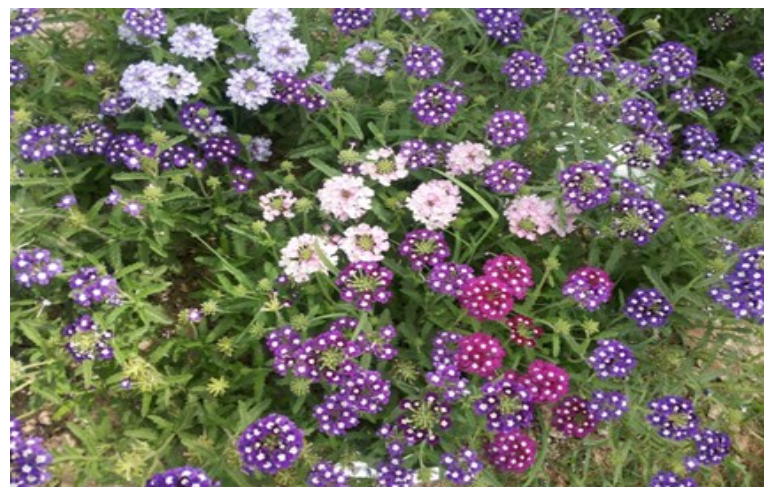

Lobularia maritima (L.)

Plate A. Floral associations of Megachile creusa Bingham, 1898.

vous; legs dorsally white.

Head: Clypeus bulging, outcurved medially, 3 dentate mandible; punctured closely, supraclypeus swollen, sculptured; hypostome declivous below antennal margin; face, clypeus and paraocular area heavily pubescent with golden down. Head wider than thorax; emarginated, quadrate

Mesosoma: Scutum protuberant and arched, outcurved, punctured closely and finely at sides, coarsely in the centre; scutellum with fulvous posterior margin, rounded posteriorly; wings flavohyaline with apical margins fuscecent; tibiae with pale white pubescence dorsally; fulvous ventrally. Metasoma: Metanotum parallel in profile, punctate finely; first two abdominal segments fulvous; tergum 6 wider than long, apical fasciae on terga 3-5 with white erect down; sterna 2-5 depressed medially, gradulii linear and complete, sterna 3-5 with white pubescence, apically rounded with dense fringe of hairs; coxal spine erect and elongate; apices of tarsi uncovered apically; tergum 6 spinose; complete and linear fasciae on sterna 2-5.

External genitalia: Gonobase thick, opaque; gonocoxites and gonostylus straight, narrow without much demarcation; long tuft of hairs present along gonocoxites and gonostylus. Penis valves narrow, straight, and parallel with hairs present on inner side. Volsella present as a triangular area.
Material examined: $2 \hat{\jmath}$, 19.iii.2015, Jalandhar $\left(31.3260^{\circ} \mathrm{N}, 75.5762^{\circ} \mathrm{E}\right) ; 10^{\lambda}$, 2.iv. 2015, Parwanoo $\left(30.8372^{\circ} \mathrm{N}, 76.9614^{\circ} \mathrm{E}\right) ; 1{ }^{\lambda}$, 24.iv. 2016, Hisar $\left(29.1492^{\circ} \mathrm{N}, 75.7217^{\circ} \mathrm{E}\right) ; 1 \mathrm{O}^{\lambda}$, 28.iv. 2016 Hisar $\left(29.1492^{\circ} \mathrm{N}, 75.7217^{\circ} \mathrm{E}\right)$.

Distribution in India: Gujarat, Rajasthan, Punjab, Harayana, Uttarakhand, Himachal Pradesh

Distribution elsewhere: Sri Lanka, Pakistan, Nepal, Myanmar

Floral associations (Plate B): Rosaceae: Prunus domestica L.; Lamiaceae: Vitex negundo L.; Lythraceae: Lagerstromia indica L.; Rosaceae: Rosa indicaL.; Fabaceae: Melilotus indica L.

Remarks: Smith originally described it as Megachile rufipes in 1858 followed by Dalla Torre in 1894 who renamed it as Megachile budhae. Bingham (1897) re-described it in "The Fauna of British India" with the same nomenclature Megachile budhae.

\section{Megachile (Pseudomegachile) elfrona Camer-} on, 1908

Megachile elfrona Cameron 1908, Entomologist. Vol. 41, p.88.

Female (Plate 4, Figs. a-e)

Diagnosis: Integument black, apices of flagellum, tarsi, claws with yellowish tinge; white pubescence on face, gena, clypeus, paraocular area, legs dorsally, tergal fascea and scopa laterally; scopa medially tinged with rufous; ventrally tarsi golden. 

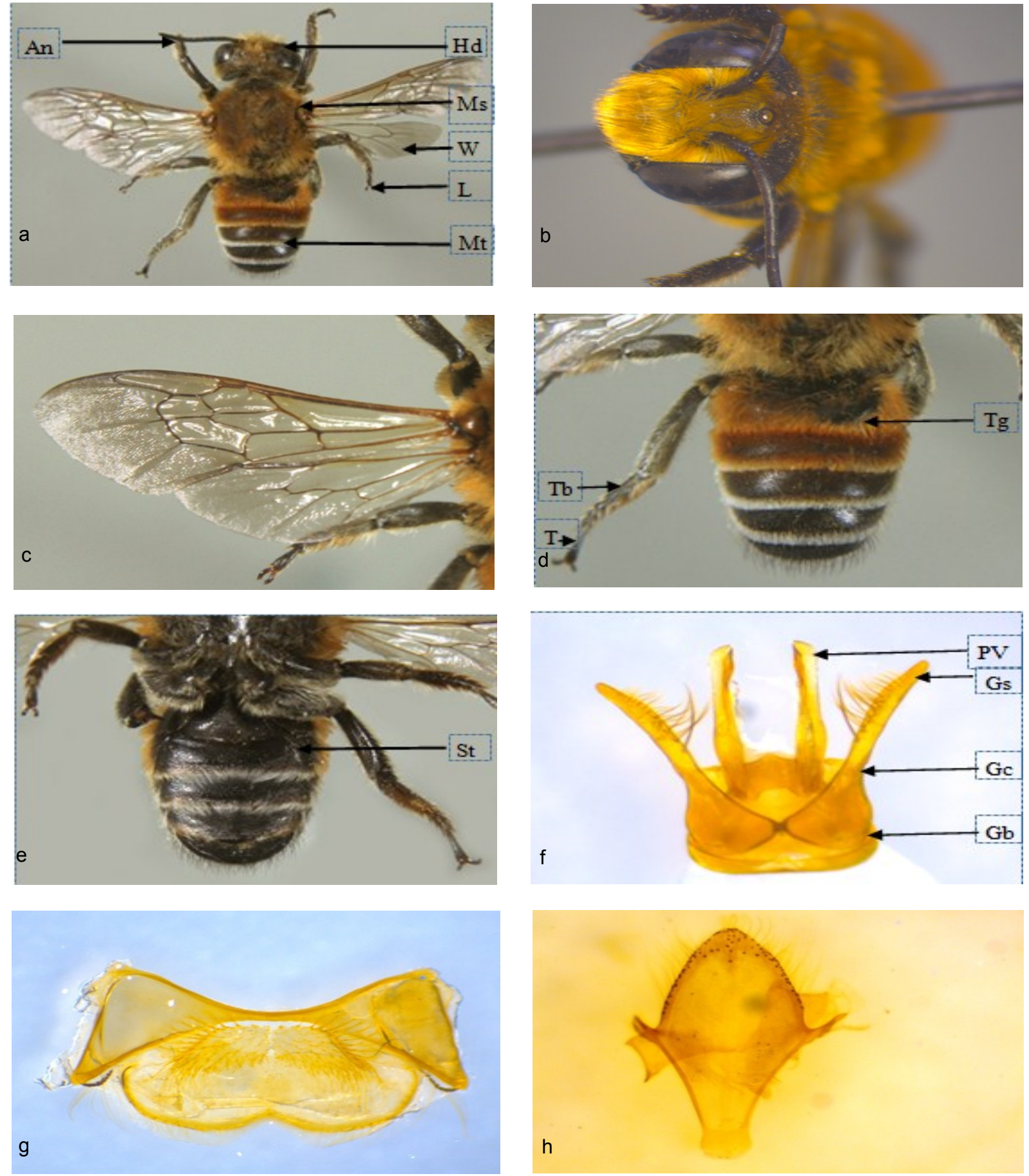

Plate 3. Megachile buddhae Dalla Torre male (a) Habitus, dorsal view (b)Head and Mesosoma, dorsal view (c) Wing (d) Metasoma, dorsal view (e) Sterna, ventral view (f) Genitalia (g) Sternum 5 (h) Sternum 8

Head: Clypeus raised, apically smooth and simple, punctured coarsely and minutely; supraclypeus strongly convex, sculptured sparsely; paraocular surface sloping below level of antennae; subocellar surface shining, convex, punctate; vertex protuberant broadly, punctured sparsely and coarsely; lateral ocelli nearer eye and occipital margin than to each other and equidistant to eye and occipital margin; narrow genae; eyes wider than genae; mandibles 4 dentate with no cutting edges.

Mesosoma: Scutum arched, reticulated punctures; median parasidal lines present; pronotal lobes slightly raised; mesepisterna closely and finely punctured; posterior margin of scutellum rounded and covered with dense, long, erect white 


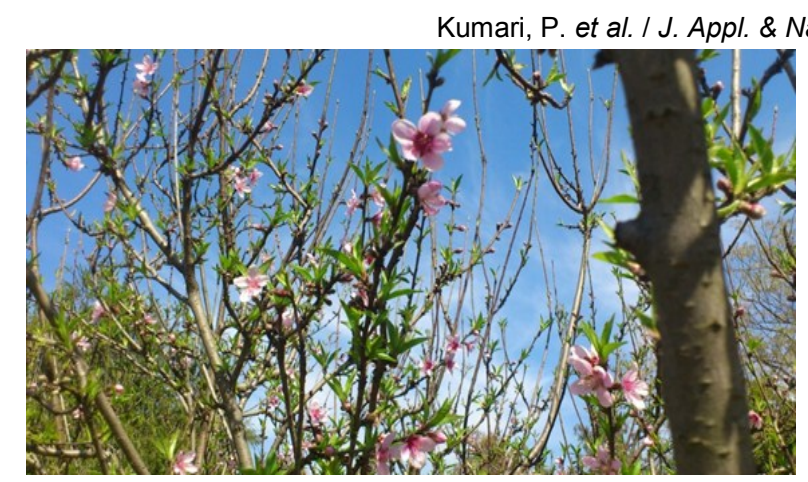

Prunus domestica L.

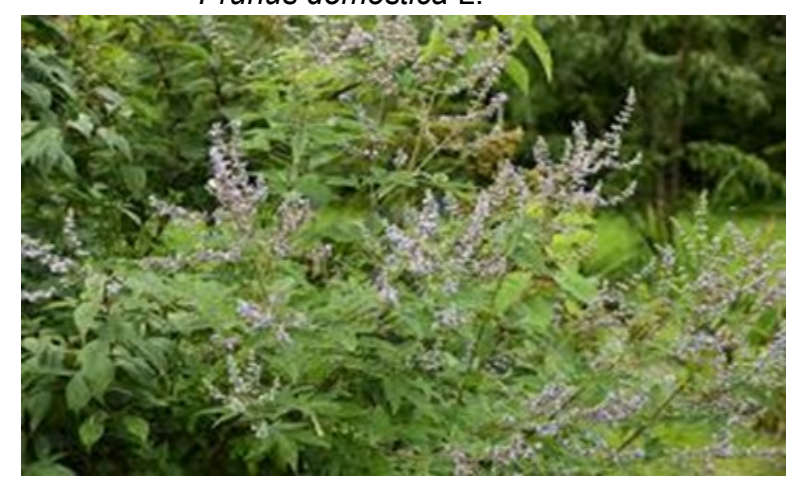

Vitex

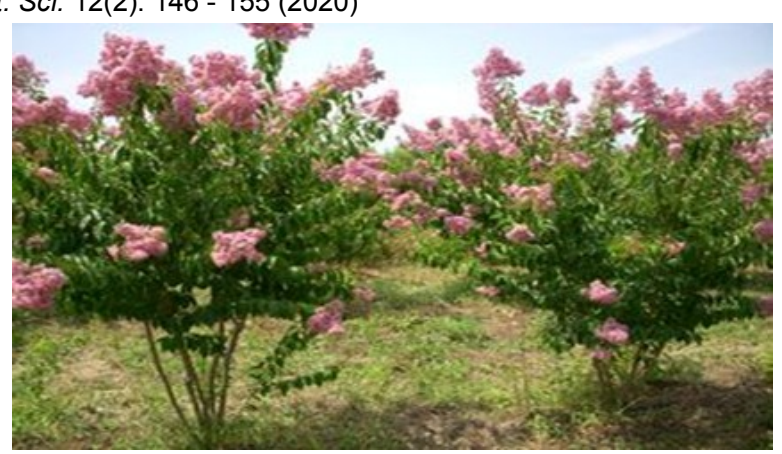

Lagerstromia indica L.

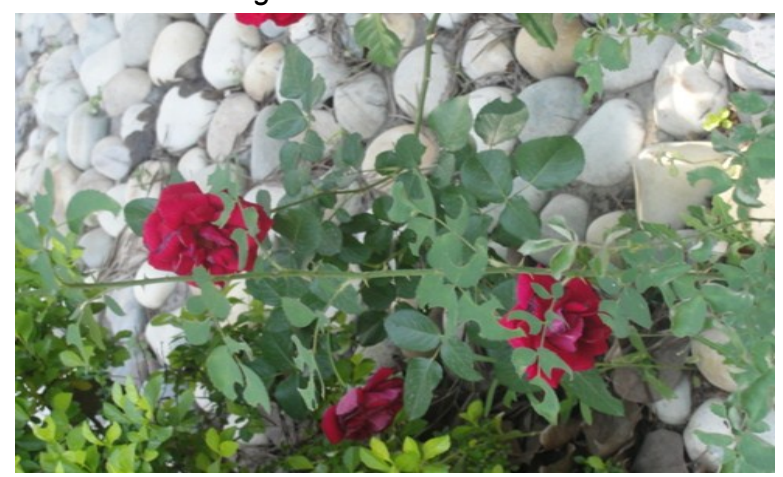

Rosa indica L.

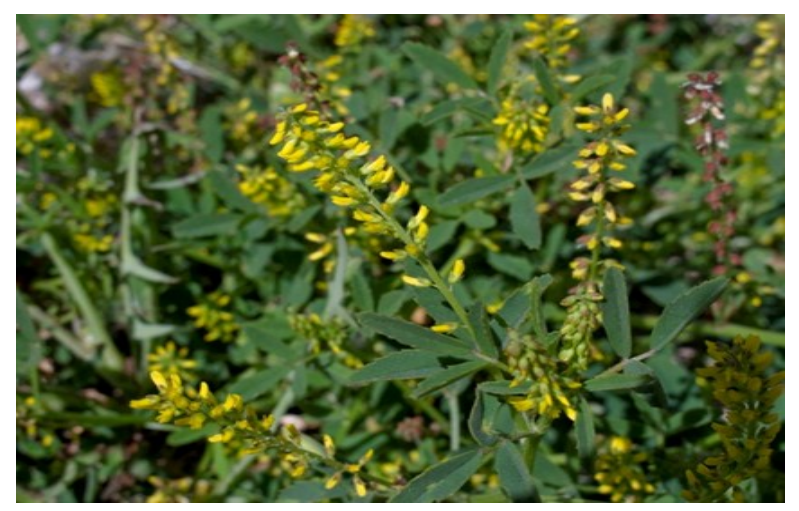

Melilotus indica L.

Plate B. Floral associations of Megachile buddhae Dalla Torre, 1894.

hairs; posterior margin of propodeal triangle recurved, apex acute; wings hyaline, veins brownish; tegulae closely and minutely punctured; legs simple, fore and hind tibiae with a short acute spine apico-medially; claws with bristles.

Metasoma: Incarinate basal tergal concavity; pregradular area shining, smooth along upper margin; finely punctured; postgradular area swollen lightly; terga 2-5 gradulii complete, linear, apical fasciae covered with white hairs; tergum 6 wider than long, concave in profile, densly punctured; sterna elevated in the middle, declivous at sides, sternum 6 transversely convex, punctured, with a short spine.

Specimen examined: 1\%, 26.iii.2014, Jalandhar $\left(31.3260^{\circ} \mathrm{N}, 75.5762^{\circ} \mathrm{E}\right) ; 1$ 1 9 , 2.iv.2014, Una $\left(31.4684^{\circ} \mathrm{N}, 76.2708^{\circ} \mathrm{E}\right) ; 2$; 9 , 13.iv.2015,Una $\left(31.4684^{\circ} \mathrm{N}, 76.2708^{\circ} \mathrm{E}\right) ; 4$; 9 , 19.iv.2015, Jalan- dhar $\left(31.3260^{\circ} \mathrm{N}, 75.5762^{\circ} \mathrm{E}\right)$.

Distribution in India: Rajasthan, Madhya Pradesh, Gujarat

Distribution elsewhere: South China, Thailand, and Indonesia

Floral associations (Plate C): Lythraceae: Punica granatum L..; Convolvulaceae: Ipomea palmatus L.

Remarks: Cameron (1908) described $M$. elfrona originally. From India, this species has been reported from Rajasthan, Madhya Pradesh and Gujarat. The present distribution of Megachile (Pseudomegachile) elfrona from Satluj Basins is new record and extended distribution of it from this area.

The above discussed species of Megachile have identical looking species from which they can be distinguished or separated on the basis of im- 
Kumari, P. et al. / J. Appl. \& Nat. Sci. 12(2): 146 - 155 (2020)
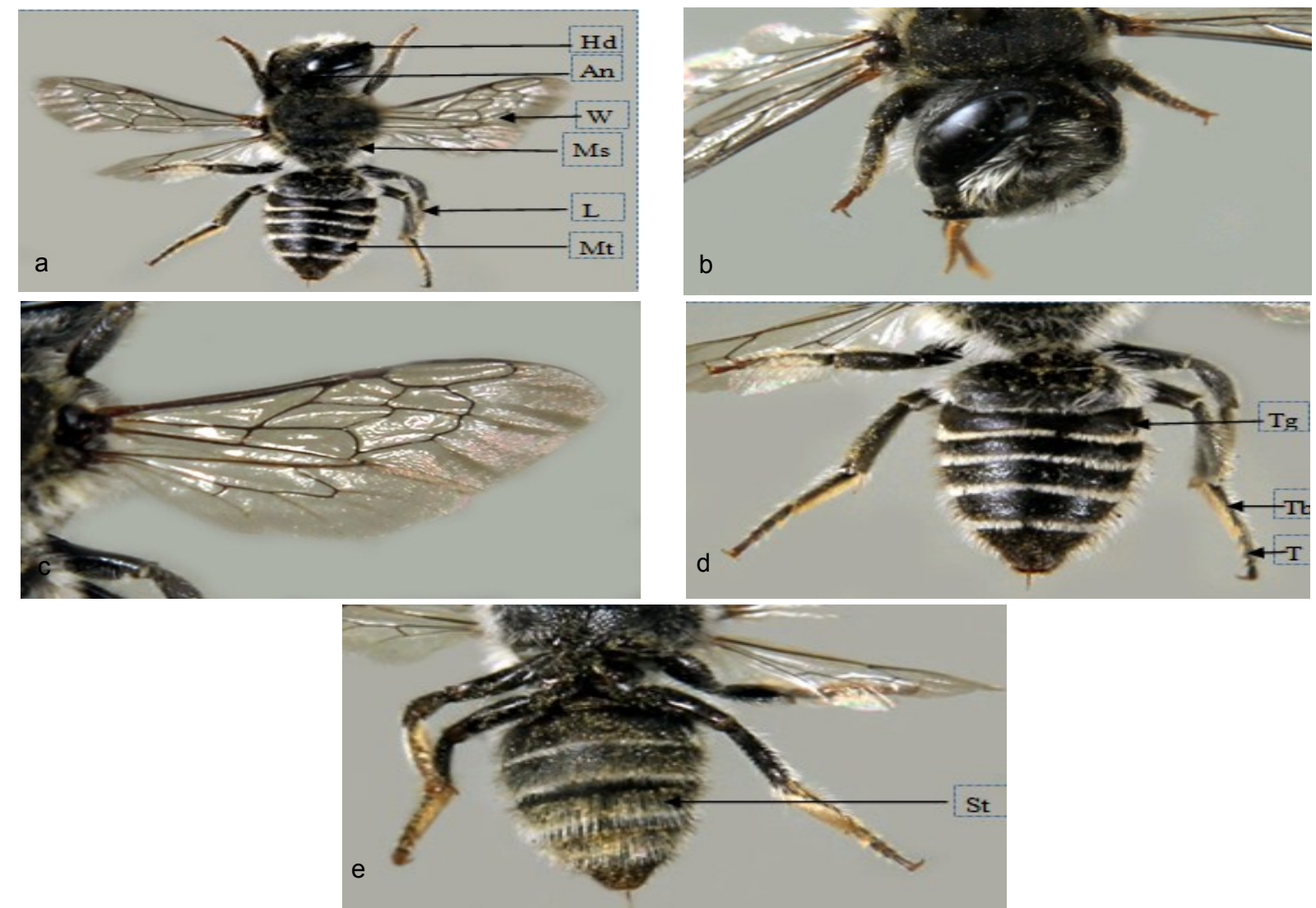

Plate 4. Megachile elfrona (Cameron) female (a) Habitus, dorsal view (b) Head and Mesosoma, dorsal view (c) Wing (d) Metasoma, dorsal view (e) Sterna, ventral view

Abbreviations used in plates : An- Antennae; Gc- Gonocoxite; Gb-Gonobase; Gs- Gonosternite; Hd- Head; Ms- Mesosoma; L- Leg; Mt- Metasoma; Md-Mandible; PV- Penis valves;T- Tarsomere; Tb- Tibia; Tg- Tergum; St- Sternum; W-Wing

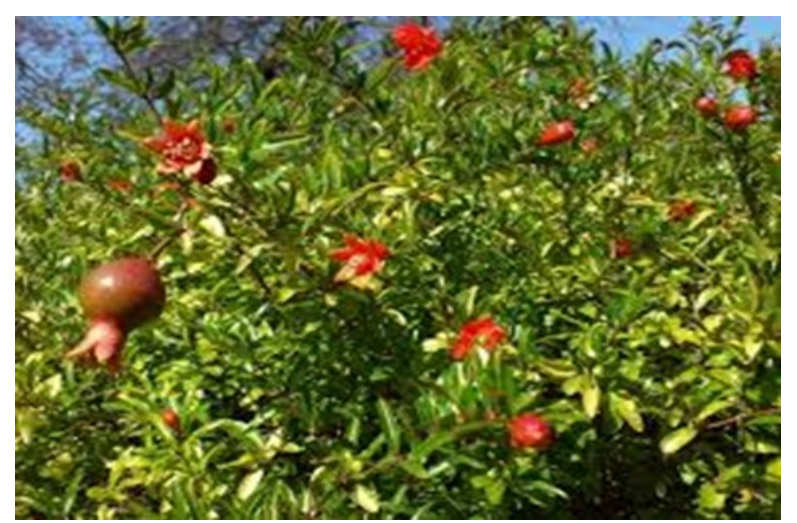

Punica granatum L.

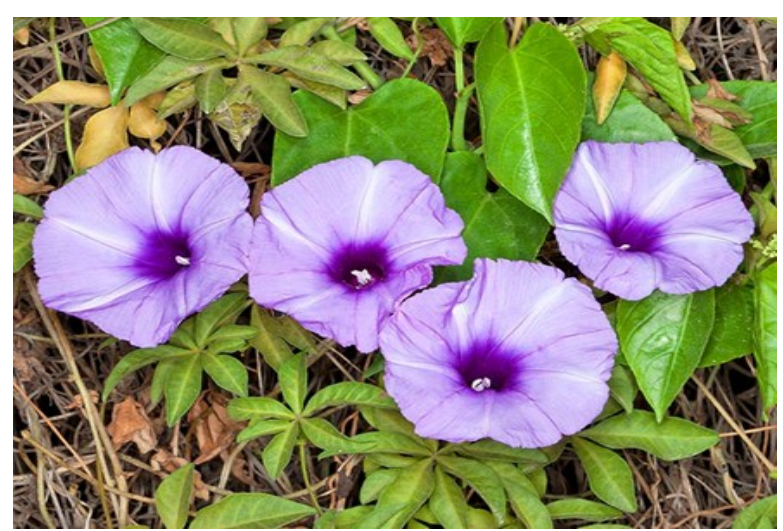

Ipomea palmatus L.

Plate C. Floral associations of Megachile) elfrona Cameron, 1908.

portant taxonomical characters. Megachile elfrona is very close to $M$. ramakrishnae Cockerell but can be distinguished by tarsi of mid and hind legs pubescence and pollen brush colour as it is orange in $M$. ramakrishnae and white in $M$. elfrona. In addition scutum is with strongly elevated medial line in $M$. ramakrishnae which is missing in $M$. elfrona. M. buddhae looks very similar to $M$. lanata but can be differentiated by the shape of abdomen. M. buddhae has broad and parallel sided abdomen whereas $M$. lanata has long and cordate abdomen. Tergum 6 is incurved in $M$. lanata and not incurved in M. buddhae.

\section{Conclusion}

The detailed survey collections from the Sutlej basin was done with some areas explored for the first time for studying Megachile species. Detailed 
descriptions of head, mesosoma and metasoma with their respective plates are illustrated, which helps in giving a good amount of information about these species. Genitalic studies help to differentiate them from similar looking species as well. As many areas of Sutlej basin were surveyed for the first time, all species were new records for these areas and will be helpful to understand the biodiversity of the species.

\section{ACKNOWLEDGEMENTS}

The authors are thankful to Director, Zoological Survey of India, Kolkata for the help in accomplishing this work.

\section{REFERENCES}

1. Bingham, C.T. (1897). Hymenoptera Wasps and bees, In: Blandford, W.T. (Ed.), The Fauna of British India, including Ceylon and Burma. Vol.1. Taylor \& Francis, London, pp. 1-579.

2. Bzdyk, E.L. (2012). A revision of the Megachile subgenus Litomegachile with an illustrated key and de- scription of a new species (Hymenoptera: Megachilidae, Megachilini). Zookeys, 221: 31-61.

3. Gupta, R.K. (1999). Taxonomic studies on the Megachilidae of North Western India. ScientificPublisher, India, 294 pp.

4. Kumari, P., Kumar, N.R., Kaur, A. and Chandra, K. (2019). Taxonomic studies on species belonging to subgenus Eutricharaea Thomson of the genus Megachile (Hymenoptera: Megachilidae). Journal of Applied and Natural Science, 11(3): 612- 618.

5. Michener, C.D. (2007). The Bees of the World. The Johns Hopkins Universitiy Press, Baltimore, USA, 972pp.

6. Michener, C.D. (2010). The Bees of the World. The Johns Hopkins Universitiy Press, Baltimore,USA, 953pp.

7. Praz, C. J. (2017). Subgeneric classification and biology of the leafcutter and dauber bees (genus Megachile Latreille) of the western Palearctic (Hymenoptera, Apoidea, Megachilidae). Journal of Hymenoptera Research, 55: 1-54.

8. Raw, A., (2007). An annotated catalogue of the leafcutter and mason bees (genus Megachile) of the Neotropics. Zootaxa, 1601, 1-127. 